Abstract: We have developed a linearly polarized Ytterbiumdoped fiber ring laser with a single longitudinal mode output at $1064 \mathrm{~nm}$. A fiber-coupled intracavity phase modulator ensured mode-hop free operation and allowed fast frequency tuning. The fiber laser was locked with high stability to an iodine-stabilized laser, showing a frequency noise suppression of a factor $\sim 10^{5}$ at $1 \mathrm{mHz}$.

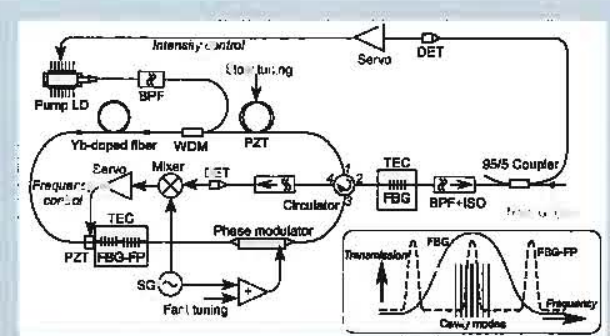

Configuration of single-mode mode-hop-free Yb-doped fiber ring laser with intracavity modulator

\title{
Experimental performance of a single-mode Ytterbium-doped fiber ring laser with intracavity modulator
}

\author{
Kenji Numata, ${ }^{1,2, *}$ Jordan Camp ${ }^{2}$ \\ ${ }^{1}$ Department of Astronomy, University of Maryland, College Park, Maryland, 20742, USA \\ ${ }^{2}$ NASA Goddard Space Flight Center, Code 663, 8800 Greenbelt Rd., Greenbelt, Maryland, 20771, USA
}

Received: XXXX 2012, Revised: X XXX XXXX, Accepted: X XXX XXXX

Published online: $\mathrm{XXXX}$ XXXX

Key words: Fiber laser, Single frequency, Frequency noise, Intensity noise

PACS: 42.55.Wd, 42.60.Mi

\section{Introduction}

We report on the development of a stable Ytterbium $(\mathrm{Yb})$-doped fiber ring laser that emits a linearly polarized, single longitudinal-mode, and continuous-wave light at $1054 \mathrm{~nm}$. Our work is originally motivated by the LISA (Laser Interferometer Space Antenna) mission [1], in which picometer-level interferometry is performed in space over 1000 seconds to detect the passage of gravitational waves. Single-frequency fiber lasers have attracted interest due to their interferometric applications; compared with a traditional laser based on bulk crystal, for example the non-planar ring oscillator (NPRO) [2], the fiber laser is robust against mechanical disturbances and has a cleaner output in terms of shape and polarization. For the same reason, it can also be readily coupled to a fiber amplifier when optical amplification is required.
In the field of interferometry, $1.064 \mathrm{~nm}$ is the common wavelength choice, due to the availability of high-quality bulk optics and the traditional Nd:YAG laser source represented by NPRO. Single-frequency fiber lasers at $1064 \mathrm{~nm}$ have been commercialized in the form of distributed feedback [3], distributed Bragg reflector [4], and ring [5] configurations, and they have been replacing traditional Nd:YAG laser in some fields. Our ring laser was built solely with commercially available components, allowing flexibility of design. We achieved stable mode-hop free operation by active frequency control using an intra-cavity fiber-coupled phase modulator, which additionally enabled fast frequency tuning. We measured and suppressed the laser's frequency and intensity noise, which are important for low-frequency interferometry. Our fiber ring laser appears to have enough stability for sensing applications.

\footnotetext{
*Corresponding author: e-mail: kenji.numata@nasa.gov
} 


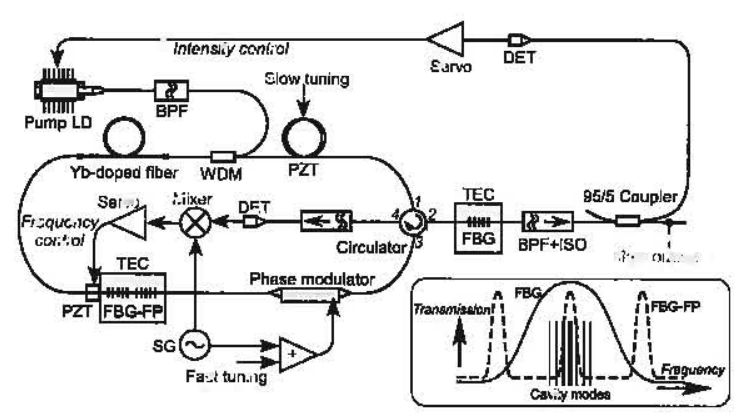

Figure 1 Ring laser configuration. WDM: wavelength division multiplexing coupler, FBG: fiber Bragg grating, FBG-FP: FBG Fabry-Perot, BPF: band-pass filter, ISO: isolator, DET: detector, TIA: transimpedance amplifier, TEC: thermo electric cooler, SG: signal generator. The light going into ports 1,2 , and 3 of the circulator comes out from ports 2,3 , and 4 , respectively.

\section{Experimental setup}

\subsection{Overview}

Figure 1 shows our ring laser configuration. The $\mathrm{Yb}$-doped gain fiber in the ring cavity was core-pumped by a laser diode (LD) through a WDM coupler. Single longitudinal mode selection was achieved by cascading an FBG and an FBG-FP filter. This configuration is similar to an earlier work done at the $1.5 \mu \mathrm{m}$ range using Erbium-doped, nonpolarization maintaining (PM) fibers [6] [7]. Using a PM $\mathrm{Yb}$ fiber and $\mathrm{PM}$ components, our ring laser produces a linearly polarized output at $1064 \mathrm{~nm}$.

\subsection{Filters and mode selection}

The inset of Fig. 1 illustrates how a single longitudinal mode is selected in this setup. We used FBG as a coarse filter to select the lasing wavelength out of Yb's wide gain bandwidth $(\sim 100 \mathrm{~nm})$. FBG was written on the slow axis of a PM980 fiber, and its peak reflectivity was about $70 \%$. The center wavelength of its reflection was specified to be at $1064.5 \mathrm{~nm}$ at room temperature, and the $3 \mathrm{~dB}$ reflection bandwidth was $0.06 \mathrm{~nm}(16 \mathrm{GHz})$. FBG was spliced to one of the four ports of the circulator so that it was used in reflection mode.

FBG-FP was used as the second filter to select one of the longitudinal modes within the FBG bandwidth. In FBG-FP, an FP cavity is formed between two FBGs written next to each other. The free spectral range (FSR) and $3 \mathrm{~dB}$ bandwidth of the FP cavity were $12 \mathrm{GHz}$ and $60 \mathrm{MHz}$, respectively. FBG-FP's $12 \mathrm{GHz}$ FSR restricted the lasing to the center of the FBG bandwidth of $16 \mathrm{GHz}$. The $60 \mathrm{MHz}$ FBG-FP's BW then selected one longitudinal mode of the $4 \mathrm{~m}$ laser cavity, whose FSR was about $51 \mathrm{MHz}$.
The unidirectional operation of this fiber ring laser, which is important to suppress instability due to spatial hole burning, was achieved by the circulator. The main laser output was taken from the transmission of the FBG after a band-pass filter isolator, which filtered out the amplified spontaneous emission (ASE). Polarization parallel to the fast axis was blocked in the circulator and isolators. The temperatures of the FBG and the FBG-FP were actively controlled and stabilized in a copper block by a thermo-electric cooler (TEC) for coarse wavelength tuning and for a stable operation.

\subsection{Control systems}

An overlap of the lasing longitudinal mode and FBG-FP resonance was actively maintained to ensure single longitudinal mode oscillation. We utilized the Pound-DreverHall technique [8] to lock FBG-FP to the laser ring cavity. The reflected light from FBG-FP is passed though an intra-cavity lithium-niobate phase modulator that phasemodulates the light at $80 \mathrm{MHz}$, and is directed through the circulator to a fiber-coupled detector. The detected signal is demodulated using a mixer. Once lasing is achieved, the demodulated signal at the mixer represents the difference between the laser frequency and the FBG-FP resonance. The signal was filtered after a proper offset was added, and it was fed back to a piezoelectric actuator (PZT) that stretches FBG-FP, forcing it to follow the laser frequency. The control bandwidth of this frequency control loop was about $1 \mathrm{kHz}$.

The output intensity of the laser was actively stabilized by monitoring a small portion of the main laser output and by controlling the injection current of the pump LD with a bandwidth of $\sim 100 \mathrm{kHz}$. This control bandwidth is limited by the tuning speed of the pump LD.

\subsection{Pump source and gain media}

The pump LD was a single-mode, PM, fiber-coupled laser at $976 \mathrm{~nm}$. The pump light was coupled into the cavity through a PM WDM coupler after passing through a narrow-band filter. The filter prevented the ASE and $1064 \mathrm{~nm}$ laser from reaching the pump LD, suppressing spurious lasing. The gain fiber was a double-cladding, single-mode, PM, Yb-doped fiber. We used it as a singleclad fiber, pumping its $6 \mu \mathrm{m}$ core. The small signal absorption of the core was $1200 \mathrm{~dB} / \mathrm{m}$ at $976 \mathrm{~nm}$, and the length of the gain fiber was about $40 \mathrm{~cm}$.

\section{Experimental results}

\subsection{Output optical power and spectrum}

Figure 2 shows the relationship between the pump power and the main laser output power. The excessive insertion 


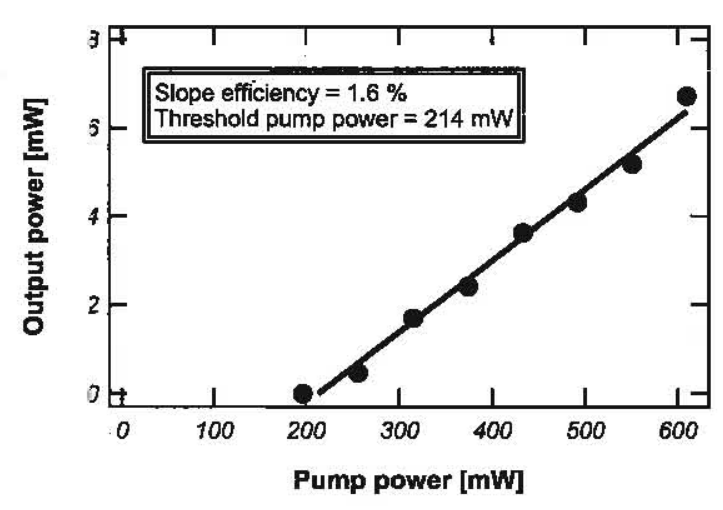

Figure 2. Laser output power dependence on pump power.

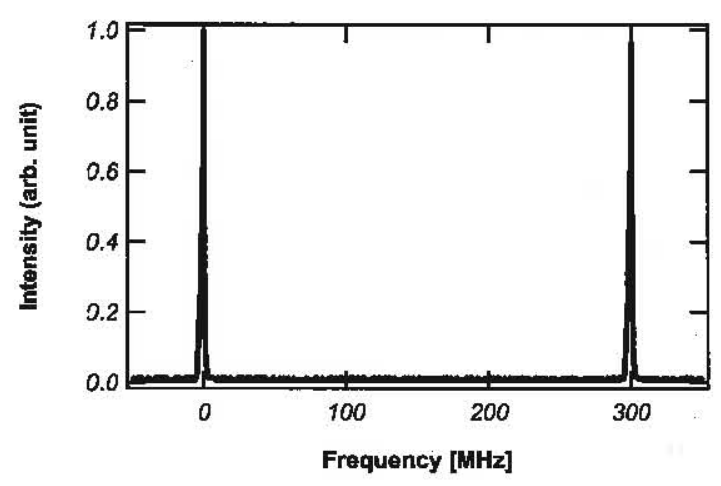

Figure 3 Output optical spectra measured by a $300 \mathrm{MHz}$ FSR scanning FP.

loss in the FBG-FP ( $4.2 \mathrm{~dB})$, the phase modulator $(2.6 \mathrm{~dB})$, the circulator $(4.9 \mathrm{~dB})$, and the filter-isolator $(1.9 \mathrm{~dB})$ resulted in a high pump threshold of $\sim 200 \mathrm{~mW}$ and an output power of $\sim 6 \mathrm{~mW}$ under a maximum available pump power of $\sim 600 \mathrm{~mW}$. The output polarization extinction ratio was better than $20 \mathrm{~dB}$.

Figure 3 shows the detailed optical spectrum measured by a scanning FP cavity with a $300 \mathrm{MHz}$ FSR. The fiber laser oscillates in single longitudinal mode, and the spectrum linewidth is below $1 \mathrm{MHz}$ (resolution limited). Single-mode lasing was maintained over several days by the frequency control loop.

\subsection{Frequency tuning}

Coarse wavelength (frequency) tuning was achieved by changing the FBG-FP spacing and FBG temperature as shown in Fig. 4. FBG-FP spacing was changed by varying the voltage applied to the PZT on the FBG-FP. The temperature was tuned between $18^{\circ} \mathrm{C}$ and $34^{\circ} \mathrm{C}$, resulting

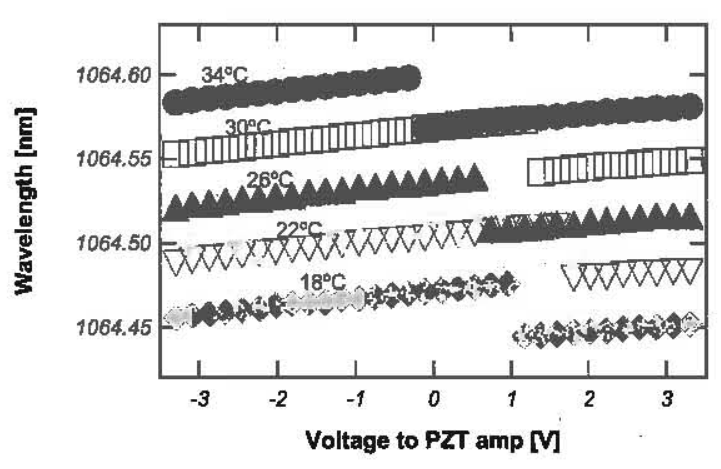

Figure 4 Wavelength tuning by FBG temperature and FBG-FP spacing. The spacing is controlled by applying a voltage onto the amplifier for the PZT that stretches the FBG-FP.

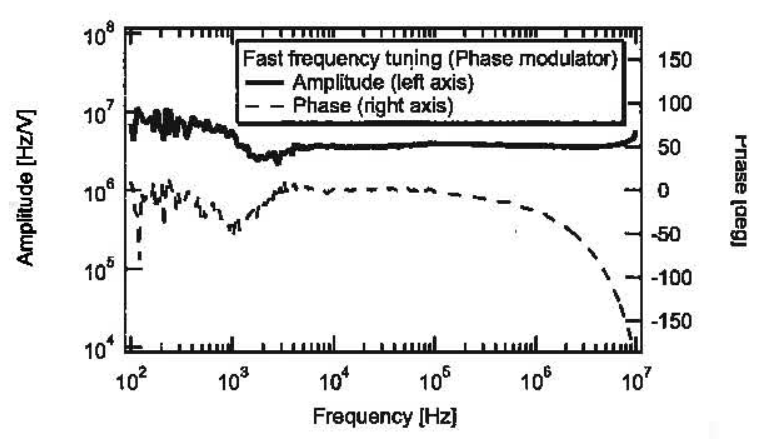

Figure 5 Response function of frequency tuning by the phase modulator.

in a center wavelength shifting of $0.25 \mathrm{~nm}(66 \mathrm{GHz})$. The wavelength jump corresponds to the FSR of the FBG-FP $(12 \mathrm{GHz})$.

When the frequency control servo is active, the laser frequency was continuously tuned by another PZT on the cavity over $4 \mathrm{GHz}$ (minimum). The cavity PZT functions as the slow but wide frequency actuator below the $1 \mathrm{kHz}$ frequency loop bandwidth.

The optical length of the cavity can also be fast-tuned by varying the voltage applied to the phase modulator. (A similar frequency tuning method in fiber ring laser can be found in [9].) We merged the modulation signal and the fast tuning signal with a wide-bandwidth operational amplifier. As shown in Fig. 5, the transfer function amplitude of such frequency tuning remains flat up to $\sim 10 \mathrm{MHz}$.

\subsection{Frequency and intensity noise}

Figure 6 shows the frequency noise spectrum of our fiber laser compared with that of a commercial NPRO laser. Below $1 \mathrm{kHz}$, our fiber laser had a comparable frequency 


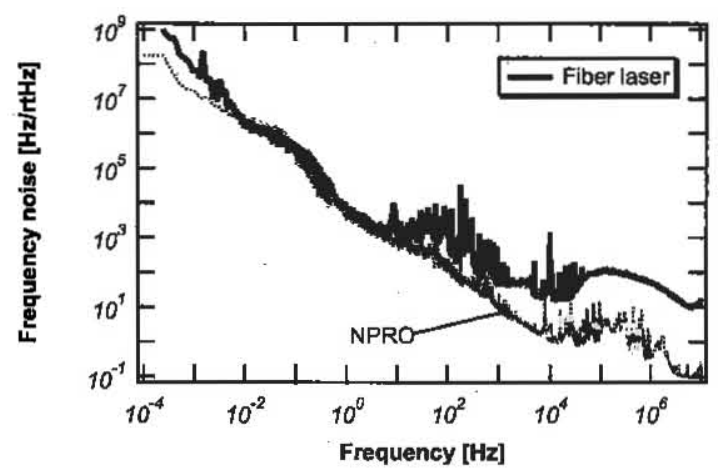

Figure 6 Frequency noise spectrum of the fiber laser. The frequency noise of commercial NPRO is also plotted for comparison.

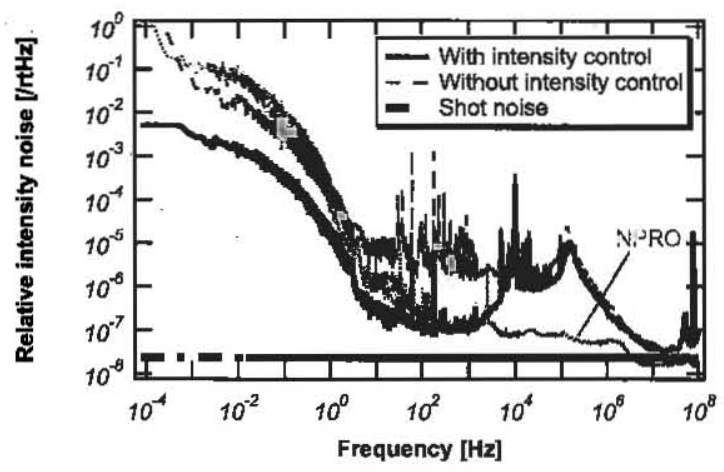

Figure 7 Relative intensity noise spectra of the fiber laser with and without the active intensity control. The intensity noise of commercial NPRO is also plotted for comparison. The shot noise level is indicated for the fiber laser at $\sim 3 \mathrm{~mW}$ detection power level.

noise to the NPRO. The noise peaks around $100 \mathrm{~Hz}$ are due to acoustic noise, which could be suppressed by proper enclosure. The excess noise (larger drift) below $10 \mathrm{mHz}$ is due to the change in overall cavity length, whose temperature is not stabilized. Above $1 \mathrm{kHz}$, our fiber laser had a larger frequency noise than the NPRO, which was in part due to relaxation oscillation.

Figure 7 shows the relative intensity noise (RIN) spectra of the fiber laser, both in free-running and stabilized conditions, and the NPRO, whose intensity is controlled by the internal servo (so-called "noise eater"). The fiber laser had a smaller RIN below $1 \mathrm{~Hz}$. However, above $10 \mathrm{kHz}$, our fiber laser had a larger intensity noise due to the relaxation oscillation at $\sim 130 \mathrm{kHz}$, which is typical for $\mathrm{Yb}$ fiber lasers (see for example [10]). The narrow intensity tuning bandwidth of the pump LD prevented the intensity servo from suppressing the RIN peak.

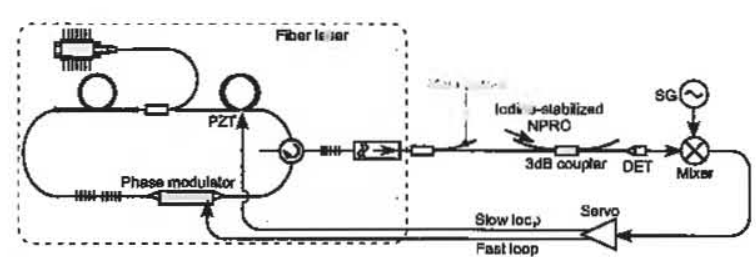

Figure 8 Setup for frequency stabilization of the fiber laser by phase-locking to a more stable laser. Only essential components are shown for the fiber laser.

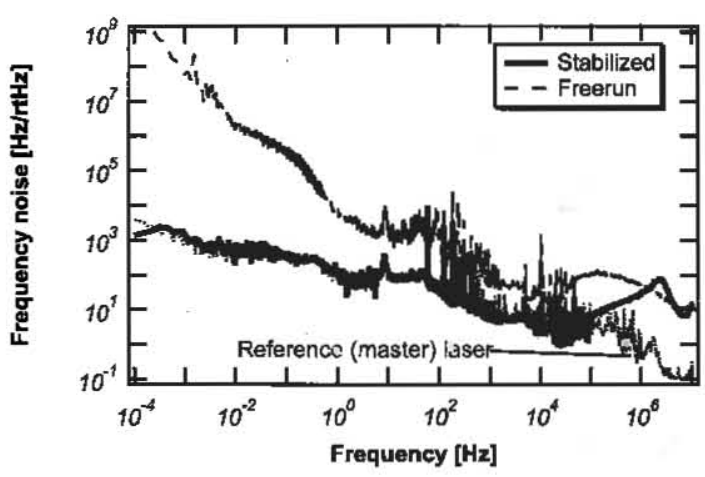

Figure 9 Frequency noise spectra of the fiber laser with and without frequency stabilization. The noise level of the reference laser, to which the fiber laser was phase-locked, is also shown.

\subsection{Frequency stabilization}

The fiber laser was phase-locked to an iodine-stabilized NPRO using slow- and fast-frequency actuators in order to demonstrate the stability and controllability of the fiber laser. Figure 8 shows the setup for the stabilization experiment. The optical offset frequency, set by the signal generator, was $100 \mathrm{MHz}$. The beatnote between the fiber laser and the iodine-stabilized NPRO was demodulated by the mixer at this frequency and was fed back to the fiber laser through the two control loops. Figure 9 shows the frequency noise of the frequency-stabilized fiber laser. The noise was suppressed within the control bandwidth of $\sim 2 \mathrm{MHz}$, and it reached the noise of the master NPRO below $\sim 80 \mathrm{kHz}$. The noise suppression factor was $\sim 10^{5}$ around $1 \mathrm{mHz}$.

\section{Discussion}

\subsection{Performance features}

Through the experiments described in the previous section, the following performance features are demonstrated: 
-The frequency and intensity noise of the fiber laser are equivalent to those of NPRO (best-known low-noise laser to date) below $\sim 1 \mathrm{kHz}$.

-The frequency noise at a higher frequency can be mitigated by the fast-frequency actuator and by locking to a frequency reference, a common technique used in precision measurement. A slow-frequency actuator allows stable frequency locking over long timescales.

-The unsupressed large relaxation oscillation peak prohibits the RIN from reaching the shot noise limit above $\sim 1 \mathrm{MHz}$, a commonly used frequency for modulation in heterodyne interferometric measurement. A better active intensity control would be required to perform shot-noise limited operation in precision measurement.

\subsection{Space applications}

For possible space use, we have performed gamma radiation tests of the optical components used in fiber laser. The result showed that fiber laser would operate in space environment without significant performance degradation. Together with the high stability and tuning capability, it makes this design a potential candidate for precision space metrology applications. Potential disadvantages of this design for space use are: 1) Many components are required. 2) The optical efficiency is low due to lossy components. 3) Noise performance at high frequency is not good as NPRO. However, a practical advantage of this design is that it can easily be rebuilt as higher performance components become available, especially a lower loss FBG-FP, phase modulator, and circulator. We will keep investigating space applications of optical-fiber technology, including lasers with active gain fiber and a new type of semiconductor laser with waveguide [11].

\section{Summary}

We developed a stable single-mode fiber ring laser at $1064 \mathrm{~nm}$. Our fiber laser offers comparable frequency and intensity noise to an NPRO, and can be frequencystabilized to the standard iodine absolute reference. It also offers fast frequency tuning capability, high polarization extinction ratio, and open architecture in which all optical components are commercially available. We think that this work is an important initial step for space fiber laser, and that it will also be useful for general laboratory applications in which stability is important.

\section{References}

[1] P. Bender, K. Danzmann, and the LISA Study Team, "Laser interferometer space antenna for the detection of gravitational waves, pre-Phase A report," Tech. Rep. MPQ233, Max-Planck-Institut für Quantenoptik, Gärching (1998). 2nd ed.
[2] T.J. Kane and R.L. Byer, Opt. Lett. 10, $65-67$ (1985).

[3] NKT Photonics, http://www.nktphotonics.com.

[4] NP Photonics, http://www.npphotonics.com.

[5] Orbits Lightwave, http://www.orbitslightwave.com.

[6] H. Inaba, A. Onae, Y. Akimoto, T. Kornukai, and M. Nakazawa, J. Quantum. Electron. 38, 1325-1330 (2002).

[7] X.P. Cheng, P. Shum, C.H. Tse, J.L. Zhou, M. Tang, W.C. Tan, R.F. Wu, and J. Zhang, Photon: Tech. Lett. 20, 976-978 (2008).

[8] R.W.P. Drever, J.L. Hall, F.V. Kowalski, J. Hough, G.M. Ford, A.J. Munley and H. Ward, Appl. Phys. B 31, 97-105 (1983).

[9] P. Ou, Y. Jia, B. Cao, C. Zhang, S. Hu, and D. Feng, Chinese Opt. Lett. 6, 845-847 (2008).

[10] S. Huang, Y. Feng, J. Dong, A. Shirakawa, M. Musha, and K. Ueda, Laser Phys. Lett. 2, 498-501 (2005).

[11] K. Numata, J. Camp, M.A. Krainak, and L. Stolpner, Opt. Exp. 18, 22781-22788 (2010). 